\title{
Research on Metal-organic Frameworks for CO2 Capture
}

$$
\text { Chunling } \mathrm{Xin}^{1, \mathrm{a}^{*} \text {, Suqing Wang }}{ }^{1, \mathrm{~b}} \text { and Yongmei Yan }{ }^{1, \mathrm{c}}
$$

${ }^{1}$ Department of Chemsitry and Chemical \& Environmental Engineering, Weifang University, Weifang 261061, China

axinchunling0925@126.com, ${ }^{b}$ wsuqing66@163.com

c sdyanyongmei@163.com, * The corresponding author

Keywords: CO2 adsorption; Metal-organic frameworks; Breakthrough curves

\begin{abstract}
Metal-organic frameworks are a new class of hybrid materials emerged in the two decades. Due to its porous structures, it is potential in the CO2 adsorption area. In this paper, we summarize the advance in metal-organic frameworks for $\mathrm{CO} 2$ capture in the five years.
\end{abstract}

\section{Introduction}

Metal-organic frameworks (MOFs), also known as coordination polymers, are novel family of porous materials constructed by coordinate bonds between organic ligands and metal atoms or metal clusters. Owing to their ultra large surface area and porosity, high crystallinity with well-defined pore properties and easily tunable structure and chemical functionality, MOFs are attracting increasing attention in the field of $\mathrm{CO}_{2}$ adsorption and separation over the past two decades.

At high pressure condition, it appears that the strong positive correlation between $\mathrm{CO}_{2}$ adsorption capacity and the surface area owing to the efficient packing and close approach of the guest molecules on the pore surface. For example, MOF-210 with a specific area of $6420 \mathrm{~cm}^{2}$ has $16.9 \mathrm{mmol} / \mathrm{g}$ of $\mathrm{CO}_{2}$ adsorption capacity at 50 bar and $298 \mathrm{~K}[1]$. The $\mathrm{CO}_{2}$ adsorption capacity of MOF-177 with a specific area of $4500 \mathrm{~m}^{2}$ reach $13.8 \mathrm{mmol} / \mathrm{g}$ at $50 \mathrm{bar}$ and $298 \mathrm{~K}$. However, the $\mathrm{CO}_{2}$ uptake decrease dramatically with the pressure, e.g. the $\mathrm{CO}_{2}$ uptake of MOF-177 is 1.48 $\mathrm{mmol} / \mathrm{g}$ at $1 \mathrm{bar}$ and $298 \mathrm{~K}$ [2]. It is demonstrated that the $\mathrm{CO}_{2}$ uptake of MOFs is related to the heat of adsorption at lower-pressure $(<1.2 \mathrm{bar})$ condition. According to the equipment of plant, the bulk of the flue gas is composed of $\mathrm{N}_{2}$ and other minor components, such as $\mathrm{H}_{2} \mathrm{O}, \mathrm{O}_{2}, \mathrm{CO}, \mathrm{NO}_{x}, \mathrm{SO}_{x}$ and so on. For post-combustion $\mathrm{CO}_{2}$ capture, the lower partial pressure of $\mathrm{CO}_{2}\left(\mathrm{P}_{\mathrm{CO} 2} \approx 0.15\right.$ bar $)$ leads to a smaller $\mathrm{CO}_{2}$ adsorption capacity. Besides, the temperature of flue gas is between 40 and $60{ }^{\circ} \mathrm{C}$. Higher temperature also results a lower $\mathrm{CO}_{2}$ adsorption capacity in MOFs. Therefore, the requirement of new-generation MOF-based adsorbent should satisfy the following requirements: a high selectivity for $\mathrm{CO}_{2}$ over other flue gas components, high adsorption uptake, lower regeneration energy consumption, long-term stability under the flue gas condition, and high adsorption rate. The $\mathrm{CO}_{2}$ affinity to the pore surface of MOFs is crucial parameter to enhance the adsorption selectivity and capacity yet minimize the regeneration energy. Current strategies to enhancing the $\mathrm{CO}_{2}$ affinity in MOFs include ligand functionalization, insertion of metal ions, framework interpenetration and incorporation of open metal sites.

\section{Ligand Functionalization}

The Incorporation of Basic Nitrogen-Containing Organic Group. The incorporation of basic nitrogen-containing organic group into MOFs is crucial for improving the $\mathrm{CO}_{2}$ adsorption selectivity and capacity. The dispersion and electrostatic forces resulting from the interaction of the quadrupole moment of $\mathrm{CO}_{2}$ with localized dipoles generated by heteroatom incorporation are typically responsible for the enhanced $\mathrm{CO}_{2}$ adsorption. The increased amount of $\mathrm{CO}_{2}$ adsorption is determined by the nature of functional groups. To date, the most studied nitrogen-functionalized ligands are heterocycle derivatives, aromatic amine derivatives, and alkylamine. There are two methods employed to incorporate nitrogen-containing groups into MOFs. One is pre-synthesis 
functionalization, namely, modifying the ligands with functional groups prior to the formation of MOFs. He et al. has done a lot of work to synthesize a series of MOFs with functionalized nitrogen-containing group[9, 12]. They designed a bent diisophthalate ligand functionalized with aminopyrimidine group and synthesized a porous ZJNU-54 via a slovothermal method. In this process, both amine group and the Lewis basic pyrimidine-ring nitrogen sites in the organic ligand are incorporated in MOFs, thus this strategy can greatly improve the selectivity of $\mathrm{CO}_{2}$. The $\mathrm{CO}_{2}$ uptake of ZJNU-54 is 120 and $189 \mathrm{~cm}^{3}$ (STP) g at $295 \mathrm{~K}$ and $278 \mathrm{~K}$ at $1 \mathrm{~atm}$, and the selectivity of $\mathrm{CO}_{2}$ with respect to $\mathrm{CH}_{4}$ is 6.1 at $298 \mathrm{~K}$ and 1 atm. The reason why ZJNU-54 exhibits a higher uptake of $\mathrm{CO}_{2}$ than $\mathrm{CH}_{4}$ is difference in the isosteric heat of adsorption. The value of isosteric heat of adsorption for $\mathrm{CO}_{2}$ and $\mathrm{CH}_{4}$ at low coverage are 24.7 and $16.8 \mathrm{~kJ} \mathrm{~mol}^{-1}$, suggesting that $\mathrm{CO}_{2}$ is adsorbed more strongly in $\mathbf{Z J N U - 5 4}$ than $\mathbf{C H}_{4}$. Another strategy is post-synthesis method, that is, to chemically modify MOFs after the formation of MOFs crystals. Choi et al.[13] have modified $\mathrm{Mg} / \mathrm{DOBDC}$ by functionalization of its open metal coordination sites with ethylene diamine (ED) to introduce pendent amines into the MOFs micropores. The result of theoretical calculation demonstrated that $1 \mathrm{ED}$ molecule was added to each unit cell. The adsorption capacity from simulated ambient air $\left(400 \mathrm{ppm} \mathrm{CO}_{2}\right)$ reached $1.51 \mathrm{mmol} / \mathrm{g}$, which is higher than that of the parent $\mathrm{Mg} / \mathrm{DOBDC}$ MOF. Besides, the adsorbent provides excellent stability over successive cycles.

The Incorporation of Other Polar Groups. Biswas et al. [14] has introduced six different functional groups (electron withdrawing, $-\mathrm{Cl},-\mathrm{Br},-\mathrm{CF}_{3}$; electron donating, $-\mathrm{CH}_{3},-\mathrm{OH},-\mathrm{OCH}_{3}$ ) into the parent MIL-47 $\left(\mathrm{V}^{\mathrm{IV}}\right)$ framework to evaluate the effect of such organic functionalities on the sorption property. The largest $\mathrm{CO}_{2}$ uptake $\left(\mathrm{mmol} \mathrm{g}^{-1}\right)$ of MIL-47-X compounds at 1 bar is MIL-47( $\left.\mathrm{V}^{\mathrm{IV}}\right)-\mathrm{OCH}_{3}$ (5.9), which is higher than that of parent MIL-47( $\left.\mathrm{V}^{\mathrm{IV}}\right)$ (4.4). ab initio DFT calculations reveal two driving forces for the enhanced $\mathrm{CO}_{2}$ affinity of the MIL-47( $\left.\mathrm{V}^{\mathrm{IV}}\right)-\mathrm{OCH}_{3}$ : (i) the presence of the substituents increases the adsorption enthalpy of $\mathrm{CO}_{2}$ and (ii) the mildly collapsed structure of the framework allows a $\mathrm{CO}_{2}$ molecule to interact simultaneously with all surrounding linkers and substituents. Recently, Huang et al.[8] have designed a keton-functionalized MOF with open $\mathrm{Cu}^{\mathrm{II}}$ sites pointing toward the cage center. The framework exhibits exceptionally high $\mathrm{CO}_{2}(46.7 \mathrm{wt} \%$ at $273 \mathrm{~K}$ and $1 \mathrm{bar})$ and displays a high selectivity of $\mathrm{CO}_{2}$ adsorption over $\mathrm{N}_{2}$ at $298 \mathrm{~K}$, which is the highest reported value under the same experimental conditions. Usage of fluorinated links to improve the $Q_{\text {st }}$ value in MOFs has caught researcher's attention in recent years. Banerjee et al.[15] have designed three isostructural MOFs from isonicotinic acid (INA) and its fluorinated analogue 3-fluoro-isonicotinic acid (FINA) along with $\mathrm{Co}^{\mathrm{II}}$ as the metal center. The $\mathrm{CO}_{2}$ adsorption results show that the fluorinated MOFs have a higher $\mathrm{CO}_{2}$ uptake (3.09 $\left.\mathrm{mmol} \mathrm{g}^{-1}\right)$.

Insertion of Metal Ions. Electrostatic force can be introduced into MOFs through the insertion of metal ions. The presence of extra metal ions can increase the electrostatic interaction with $\mathrm{CO}_{2}$ and act as additional adsorption sites[11]. Besides, the $Q_{\mathrm{st}}$ value of $\mathrm{CO}_{2}$ adsorption should be increased to a certain degree that minimized the energy penalty for regeneration. Yeganegi et al. have done theoretical study on the effect of lithium cation on the adsorption and sepearation of $\mathrm{CO}_{2}$, $\mathrm{CH}_{4}$, and $\mathrm{H}_{2}$ on a $\mathrm{Zn}_{2}(\mathrm{NDC})_{2}$ (diPyNI). The results indicated that the selectivity of $\mathrm{CO}_{2} / \mathrm{H}_{2}$ on MOF was higher than that of $\mathrm{CO}_{2} / \mathrm{CH}_{4}$ at room temperature and pressure up to 50 bar[16]. Thus, $\mathrm{Li}^{+}$ doping is a possible way for making effective MOFs for gas storage and separation. Suh. et al. have done much study on various metal ions. They impregnated $\mathrm{Li}^{+}, \mathrm{Mg}^{2+}, \mathrm{Ca}^{2+}, \mathrm{Co}^{2+}$, and $\mathrm{Ni}^{2+}$ in the channels of SNU-100, and the $Q_{\mathrm{st}}$ value of $\mathrm{CO}_{2}$ adsorption in the MOF to the range of 37.4-34.5 kJ $\mathrm{mol}^{-1}$ as well as the adsorption selectivity of $\mathrm{CO}_{2}$ over $\mathrm{N}_{2}$ to $40.4-31.0 \mathrm{~kJ} \mathrm{~mol}^{-1}$ at room temperature[11]. Among various metal ions, the $\mathrm{Ca}^{2+}$ ions seems to be the best for increasing the isosteric heat of $\mathrm{CO}_{2}$ adsorption. The $Q_{\mathrm{st}}$ value of $\mathrm{CO}_{2}$ adsorption for metal-doping MOFs is always lower than that of amine-functionalized MOFs. e. g. the isosteric heat of adsorption for mmen- $\mathrm{Mg}_{2}(\mathrm{dobpdc})$ approached a value of $71 \mathrm{~kJ} \mathrm{~mol}^{-1}[17]$.

\section{Incorporation of Open Metal Sites.}

Open meal sites in the MOFs can provide coordination bonding sites for $\mathrm{CO}_{2}$ molecules[18]. e. $\mathrm{g}$. the initial isosteric heat of adsorption is $39 \mathrm{~kJ} \mathrm{~mol}^{-1}$ for Mg-MOF-74. Hence, it is expected that 
removal of $\mathrm{CO}_{2}$ from the MOF will require less energy in the separation process. The exceptional high uptake of Mg-MOF-74 for $\mathrm{CO}_{2}$ may be attributed to the strong interactions between the oxygen lone pair orbitals of $\mathrm{CO}_{2}$ with the coordinately unsaturated metal cations[19]. Nowadays, the most researched MOFs with open metal sites are HKUST-1[20], MIL-101 series[21], and MOF-74 series[7]. The open metal sites are typically obtained by incorporating solvent molecules as terminal ligands, which can be readily removed following desolvation at elevated temperatures or under vacuum. Recently, Huang et al. have synthesized a polar ketone-functionalized metal-organic framework with exposed metal sites showing a high $\mathrm{CO}_{2}$ adsorption performance (46.7 wt \% at 273 $\mathrm{K}$ and 1 bar), which exceeds MOF-74 that known as $\mathrm{CO}_{2}$ adsorbents with the highest $\mathrm{CO}_{2}$ adsorption at lower pressure. The results suggest that implanting polar ketone groups into a multidentate ligand could throw cetain light on designing and preparing novel MOFs with high performance in the research of gas adsorption[8].

\section{Conclusion}

For post-combustion $\mathrm{CO}_{2}$ capture, the $\mathrm{CO}_{2}$ adsorption capacities of some MOFs with unsaturated metal sites or amino functional group usually reach $3 \mathrm{mmol} \bullet \mathrm{g}^{-1}$. This is because interaction between $\mathrm{CO}_{2}$ molecules and the skeleton of MOFs. The focus of application for this material is to make more functional materials. Design and synthesis of composite material with the versatility, modifying and optimizing the hole cavity of MOFs and controling their chemical stability and reactivity are the three approaches to design a more suitable for the separation of the flue gas environment $\mathrm{CO}_{2} / \mathrm{N}_{2}$ mixtures of $\mathrm{CO}_{2}$ adsorbent.

\section{Acknowledgement}

We acknowledge the financial support of Weifang University Scientific Research Fund for Doctor (No. 2016BS09) and The Development of Science and Technology Plan project in Weifang (2016GX013).

\section{References}

[1] H Furukawa, N Ko, Y B Go, et al. Ultrahigh porosity in metal-organic frameworks[J]. Science 2010, 329: 424-8.

[2] K Sumida, D L Rogow, J A Mason, et al. Carbon dioxide capture in metal-organic frameworks[J]. Chem Rev 2012, 112: 724-81.

[3] B Ugale, S S Dhankhar, C M Nagaraja. Construction of 3d homochiral metal-organic frameworks (MOFs) of $\mathrm{Cd}(\mathrm{ii})$ : Selective $\mathrm{CO} 2$ adsorption and catalytic properties for the knoevenagel and henry reaction[J]. Inorg. Chem. Front. 2017, 4: 348-359.

[4] S Pourebrahimi, M Kazemeini, L Vafajoo. Embedding graphene nanoplates into mil-101(Cr) pores: Synthesis, characterization, and $\mathrm{CO} 2$ adsorption studies[J]. Industrial \& Engineering Chemistry Research 2017, 56: 3895-3904.

[5] C Xin, H Zhan, X Huang, et al. Effect of various alkaline agents on the size and morphology of nano-sized hkust-1 for CO2 adsorption[J]. RSC Advances 2015, 5: 27901-27911.

[6] Y Jiang, L Sun, J Du, et al. Multifunctional zinc metal-organic framework based on designed H4TCPP ligand with aggregation-induced emission effect: $\mathrm{CO} 2$ adsorption, luminescence, and sensing property[J]. Crystal Growth \& Design 2017, 17: 2090-2096.

[7] D-A Yang, H-Y Cho, J Kim, et al. CO2 capture and conversion using Mg-MOF-74 prepared by a sonochemical method[J]. Energy \& Environmental Science 2012, 5: 6465.

[8] G Feng, Y Peng, W Liu, et al. Polar ketone-functionalized metal-organic framework showing a high CO2 adsorption performance[J]. Inorg Chem 2017, 56: 2363-2366. 
[9] C Song, Y Ling, L Jin, et al. CO2 adsorption of three isostructural metal-organic frameworks depending on the incorporated highly polarized heterocyclic moieties[J]. Dalton Trans 2016, 45: 190-7.

[10] B Liu, Y-H Jiang, Z-S Li, et al. Selective CO2 adsorption in a microporous metal-organic framework with suitable pore sizes and open metal sites[J]. Inorg. Chem. Front. 2015, 2: $550-557$.

[11]H J Park, M P Suh. Enhanced isosteric heat, selectivity, and uptake capacity of co2adsorption in a metal-organic framework by impregnated metal ions[J]. Chem. Sci. 2013, 4: 685-690.

[12] J Jiao, L Dou, H Liu, et al. An aminopyrimidine-functionalized cage-based metal-organic framework exhibiting highly selective adsorption of $\mathrm{C} 2 \mathrm{H} 2$ and $\mathrm{CO} 2$ over $\mathrm{CH} 4[\mathrm{~J}]$. Dalton Trans 2016, 45: 13373-82.

[13] S Choi, $\mathrm{T}$ Watanabe, $\mathrm{T}-\mathrm{H}$ Bae, et al. Modification of the $\mathrm{Mg} / \mathrm{dobdc}$ mof with amines to enhance CO2 adsorption from ultradilute gases[J]. Journal of Physical Chemistry Letters 2012, 3: 1136-1141.

[14]S Biswas, D E P Vanpoucke, $T$ Verstraelen, et al. New functionalized metal-organic frameworks MIL-47-x ( $\mathrm{x}=-\mathrm{Cl},-\mathrm{Br},-\mathrm{CH} 3,-\mathrm{CF} 3,-\mathrm{OH},-\mathrm{OCH} 3)$ : Synthesis, characterization, and $\mathrm{CO} 2$ adsorption properties[J]. The Journal of Physical Chemistry C 2013, 117: 22784-22796.

[15]P Pachfule, Y Chen, J Jiang, et al. Fluorinated metal-organic frameworks: Advantageous for higher $\mathrm{H} 2$ and $\mathrm{CO} 2$ adsorption or not?[J]. Chemistry 2012, 18: 688-94.

[16] V Sokhanvaran, S Yeganegi. Multiscale computational study on the adsorption and separation of $\mathrm{CO} 2 / \mathrm{CH} 4$ and $\mathrm{CO} 2 / \mathrm{H} 2$ on Li+ -doped mixed-ligand metal-organic framework Zn2 (NDC)2 (dipyni)[J]. Chemphyschem 2016, 17: 4124-4133.

[17] T M McDonald, W R Lee, J A Mason, et al. Capture of carbon dioxide from air and flue gas in the alkylamine-appended metal-organic framework mmen-Mg2(DOBDPC)[J]. J Am Chem Soc 2012, 134: 7056-65.

[18]D Britt, H Furukawa, B Wang, et al. Highly efficient separation of carbon dioxide by a metal-organic framework replete with open metal sites[J]. Proc Natl Acad Sci U S A 2009, 106: 20637-40.

[19]Z Bao, L Yu, Q Ren, et al. Adsorption of CO2 and CH4 on a magnesium-based metal organic framework[J]. J Colloid Interface Sci 2011, 353: 549-56.

[20] H Wang, Z G Qu, W Zhang, et al. Experimental and numerical study of CO2 adsorption on copper benzene-1,3,5-tricarboxylate $(\mathrm{Cu}-\mathrm{BTC})$ metal organic framework[J]. International Journal of Heat and Mass Transfer 2016, 92: 859-863.

[21]S Ye, X Jiang, L-W Ruan, et al. Post-combustion CO2 capture with the hkust-1 and MIL-101(Cr) metal-organic frameworks: Adsorption, separation and regeneration investigations[J]. Microporous and Mesoporous Materials 2013, 179: 191-197. 